\title{
Higgsing the stringy higher spin symmetry
}

\section{Matthias R. Gaberdiel, ${ }^{a}$ Cheng Peng ${ }^{a}$ and Ida G. Zadeh ${ }^{b}$}

\author{
${ }^{a}$ Institut für Theoretische Physik, ETH Zurich, \\ CH-8093 Zürich, Switzerland \\ ${ }^{b}$ Martin Fisher School of Physics, Brandeis University, \\ Waltham, MA 02454, U.S.A. \\ E-mail: gaberdiel@itp.phys.ethz.ch, pengch@itp.phys.ethz.ch, \\ zadeh@brandeis.edu
}

ABSTRACT: It has recently been argued that the symmetric orbifold theory of $\mathbb{T}^{4}$ is dual to string theory on $\mathrm{AdS}_{3} \times \mathrm{S}^{3} \times \mathbb{T}^{4}$ at the tensionless point. At this point in moduli space, the theory possesses a very large symmetry algebra that includes, in particular, a $\mathcal{W}_{\infty}$ algebra capturing the gauge fields of a dual higher spin theory. Using conformal perturbation theory, we study the behaviour of the symmetry generators of the symmetric orbifold theory under the deformation that corresponds to switching on the string tension. We show that the generators fall nicely into Regge trajectories, with the higher spin fields corresponding to the leading Regge trajectory. We also estimate the form of the Regge trajectories for large spin, and find evidence for the familiar logarithmic behaviour, thereby suggesting that the symmetric orbifold theory is dual to an AdS background with pure RR flux.

Keywords: Higher Spin Symmetry, Conformal and W Symmetry, AdS-CFT Correspondence, Long strings

ARXIV EPRINT: 1506.02045 


\section{Contents}

1 Introduction $\quad 2$

2 The symmetric orbifold 3

2.1 The quadratic fields 4

2.2 The cubic and higher order fields 5

$\begin{array}{lll}3 & \text { The exactly marginal operators } & 6\end{array}$

$\begin{array}{lll}3.1 & \text { Exactly marginal operators in the twisted sector } & 7\end{array}$

3.1.1 The perturbing fields $\quad 8$

4 First order deformation analysis $\quad 8$

4.1 Deformation by the untwisted sector perturbation fields 9

$\begin{array}{lll}4.2 & \text { Deformation by the twisted sector perturbation fields } & 9\end{array}$

5 Second order deformation analysis $\quad 10$

5.1 A more structural approach 11

$\begin{array}{ll}5.2 \text { Relation to bulk masses } & 12\end{array}$

6 Explicit anomalous dimensions $\quad 12$

$\begin{array}{ll}\text { 6.1 The structure of the analysis } & 13\end{array}$

$\begin{array}{ll}\text { 6.2 The anomalous dimensions at low spin } & 13\end{array}$

$\begin{array}{lll}6.2 .1 & \text { Spin } 1 & 13\end{array}$

6.2.2 Spin $\frac{3}{2} \quad 13$

$\begin{array}{lll}6.2 .3 & \text { Spin } 2 & 14\end{array}$

$\begin{array}{lll}6.2 .4 & \text { Spin } \frac{5}{2} & 14\end{array}$

$\begin{array}{lll}6.2 .5 & \text { Spin } 3 & 14\end{array}$

6.3 Partial diagonalisation for higher spin 15

$\begin{array}{lll}7 & \text { Discussion } & 17\end{array}$

$\begin{array}{ll}\text { A Free field realisation of the chiral fields } & 19\end{array}$

B The second order analysis $\quad 20$

B.1 Using Stokes' theorem 20

B.2 Using separation of variables $\quad 22$ 


\section{Introduction}

Recently some progress relating the higher spin/CFT duality to the stringy AdS/CFT correspondence was made for the case of $\mathrm{AdS}_{3}$. In particular, it was shown in [1] that a certain limit of the CFT dual of the higher spin theory with $\mathcal{N}=4$ supersymmetry [2] forms a closed subsector of the symmetric orbifold theory, the CFT dual of string theory on $\mathrm{AdS}_{3} \times \mathrm{S}^{3} \times \mathbb{T}^{4}$.

Among other things, this result suggests that the symmetric orbifold theory $\left(\mathbb{T}^{4}\right)^{\otimes N} / S_{N}$ is dual to string theory at the tensionless point. At this point in moduli space, the symmetry algebra of string theory is much bigger than just the $\mathcal{N}=4$ superconformal algebra, and indeed even bigger than the vector-like symmetry algebra of the Vasiliev higher spin theory [3-5]. In fact, the usual picture one has in mind is that the Vasiliev fields correspond to the leading Regge trajectory that become massless (higher spin fields) at the tensionless point. By the same token, one should then expect that also the higher Regge trajectories lead to massless higher spin fields, and this seems indeed to be in line with the structure of the dual CFT, as studied in detail in [6].

In this paper we want to confirm, at least qualitatively, this picture by studying the perturbation of the symmetric orbifold theory that corresponds to switching on the string tension. Under this perturbation, we expect that the symmetry algebra is broken down to the $\mathcal{N}=4$ superconformal algebra. In addition, we should expect that the masses of the fields associated to the Vasiliev $\mathcal{W}_{\infty}$ algebra should be smallest, at each given spin, relative to those of the additional higher spin fields (that correspond to the higher Regge trajectories). Furthermore, one may hope that we see something like 'Regge' trajectories to emerge in this regime. Using conformal perturbation theory we will find what we believe to be very convincing evidence for this picture: to the extent to which we have managed to determine the anomalous dimensions, those of the original $\mathcal{W}_{\infty}$ algebra are indeed smallest (at a given spin). Furthermore, the additional symmetry generators of the stringy symmetry algebra seem to fall naturally into different Regge trajectories, where the $n^{\text {th }}$ trajectory corresponds to the symmetrised polynomials of $n+1$ free fields of the symmetric orbifold theory. We also find evidence that the anomalous dimensions $\gamma(s)$ behave as $\log s$ at large spin $s$, at least before any mixing effects are taken into account. This is in agreement with expectations from the analysis of classical string solutions in $\mathrm{AdS}_{5}$, see [7] and, e.g., [8] for a review, or the explicit results for $\mathrm{AdS}_{3}$, see in particular [9, 10].

The paper is organised as follows. In section 2 we explain the structure of the higher spin fields of the symmetric orbifold. Section 3 describes the exactly marginal operators that include the moduli of the underlying $\mathbb{T}^{4}$ torus, as well as the modulus from the 2 -cycle twisted sector that corresponds to switching on the string tension. In section 4 we show, by a first order calculation, that the former do not break the higher spin symmetry, whereas the latter does. In order to determine the anomalous dimensions (and hence the induced masses) quantitatively, we then turn to a second order analysis in section 5 . We also explain the relation between anomalous dimensions and bulk masses there. In section 6 we then describe the results of our explicit computations: in section 6.2 we give the exact anomalous dimensions for the low spin operators to lowest non-trivial order in the perturbation, while 
in section 6.3 we calculate the diagonal matrix elements for the quadratic (leading Regge trajectory) and cubic (first subleading Regge trajectory) generators at large spin. We discuss our results in section 7 . There are two appendices, where we give the explicit expressions for the higher spin generators in terms of the free fields (appendix A), and the integrals of the second order analysis of the perturbation calculation (appendix B). We have also included an ancillary file in the arXiv submission of this paper which contains the numerical values of the diagonal elements of the mixing matrix for the quadratic and cubic generators, as well as analytic expressions for these diagonal elements.

\section{The symmetric orbifold}

A duality between matrix extended higher spin theories on $\mathrm{AdS}_{3}$ with large $\mathcal{N}=4$ supersymmetry and the Wolf space coset models was proposed in [2], see also [11-14] for further developments of matrix extended higher spin theories on $\mathrm{AdS}_{3}$. In the limit where the large $\mathcal{N}=4$ superconformal algebra contracts to the more familiar small $\mathcal{N}=4$ algebra (and hence the higher spin theory should correspond to $\mathrm{AdS}_{3} \times \mathrm{S}^{3} \times \mathbb{T}^{4}$ ), the 'perturbative part' of the Wolf space cosets becomes a closed subsector of the symmetric orbifold $\left(\mathbb{T}^{4}\right)^{\otimes(N+1)} / S_{N+1}$, which in turn is thought to be dual to string theory on $\operatorname{AdS}_{3} \times \mathrm{S}^{3} \times \mathbb{T}^{4}$ at the tensionless point. This therefore ties in nicely with the general belief that the perturbative higher spin theory should describe a subsector of string theory in the tensionless limit.

In this section we want to describe the symmetry algebra of the symmetric orbifold (that contains in particular a $\mathcal{W}_{\infty}[0]$ algebra as a subalgebra). Before applying the orbifold projection the theory is generated by $2(N+1)$ complex bosons and $2(N+1)$ complex fermions, as well as their hermitian conjugates; we shall denote these fields by $\phi_{a}^{(i)}, \bar{\phi}_{a}^{(i)}$, $\psi_{a}^{(i)}, \bar{\psi}_{a}^{(i)}$, where $i=1,2$, and $a=1, \ldots, N+1$ labels the different copies. (Here the $\bar{\phi}_{a}^{(i)}$ are the hermitian conjugates of the $\phi_{a}^{(i)}$, and similarly for the fermions.) Their non-trivial OPEs then have the form

$$
\begin{aligned}
\partial \bar{\phi}_{a}^{(i)}(z) \partial \phi_{a^{\prime}}^{(j)}(w) & \sim \frac{\delta^{i j} \delta_{a a^{\prime}}}{(z-w)^{2}}+\mathcal{O}(1), \\
\bar{\psi}_{a}^{(i)}(z) \psi_{a^{\prime}}^{(j)}(w) & \sim \frac{\delta^{i j} \delta_{a a^{\prime}}}{(z-w)}+\mathcal{O}(1) .
\end{aligned}
$$

The orbifold group $S_{N+1}$ acts by permuting the $N+1$ copies (labelled by $a$ ). The higher spin fields come from the untwisted sector of the orbifold, and their single-particle generators are all of the form

$$
\sum_{a=1}^{N+1} P_{a}^{j_{1}} \cdots P_{a}^{j_{m}}
$$

where each $P_{a}^{j}$ is one of the 4 bosons or fermions in the $a^{\prime}$ th copy, including possibly derivatives. In this language, the higher spin fields that are dual to the Vasiliev theory are associated to the bilinear generators, i.e., to the fields (2.3) with $m=2$. Strictly speaking the original $\mathcal{W}_{\infty}[0]$ algebra of [2] corresponds only to the neutral bilinear generators, i.e., to those where we have one unbarred and one barred generator. However, it is not difficult to 
see (see also section 2.1 below) that if we extend $\mathcal{W}_{\infty}[0]$ by the generators corresponding to $(0 ;[2,0, \ldots, 0])$ and $(0 ;[0, \ldots, 0,2])$, we still obtain a Vasiliev-type higher spin algebra $[6]$.

The other generators of the stringy algebra can be organised in representations of this extended $\mathcal{W}_{\infty}$ algebra - the columns of the Higher Spin Square [6] - where we have one column for each $m=2,3,4, \ldots$, with $m=2$ corresponding to the bilinear $\mathcal{W}_{\infty}$ generators. It is then natural to believe that the higher spin fields associated to $m=2$ lie on the leading Regge trajectory, those associated to $m=3$ on the first sub-leading Regge trajectory, etc. Indeed, in flat space, the different Regge trajectories are constructed, roughly speaking, by different numbers of creation operators, and this is directly parallel to the above.

\subsection{The quadratic fields}

For the following it will be important to understand the structure of the single particle generators of the stringy algebra in a little more detail. The original $\mathcal{W}_{\infty}$ [0] algebra of [2] is generated by the $\mathcal{N}=4$ superconformal algebra together with a number of multiplets,

$$
\mathcal{W}_{\infty}[0]: \quad(\mathcal{N}=4) \oplus \bigoplus_{s=1}^{\infty} R^{(s)},
$$

where $R^{(s)}$ is the $\mathcal{N}=4$ supermultiplet consisting of the generators

$$
\begin{array}{rcc} 
& s: & (\mathbf{1}, \mathbf{1}) \\
& s+\frac{1}{2}: & (\mathbf{2}, \mathbf{2}) \\
R^{(s)}: & s+1: & (\mathbf{3}, \mathbf{1}) \oplus(\mathbf{1}, \mathbf{3}) \\
& s+\frac{3}{2}: & (\mathbf{2}, \mathbf{2}) \\
s+2: & (\mathbf{1}, \mathbf{1}) .
\end{array}
$$

Here the quantum numbers are the dimensions with respect to the $\mathfrak{s u}(2)_{+} \oplus \mathfrak{s u}(2)_{-}$algebra (that is contained in the $\mathcal{N}=4$ superconformal algebra). ${ }^{1}$ To be more specific, the $\mathcal{N}=4$ superconformal algebra contains 4 spin $s=\frac{1}{2}$ fields, that are described by the orbifold invariant combinations

$$
f^{(i)}=\sum_{a=1}^{N+1} \psi_{a}^{(i)}, \quad \bar{f}^{(i)}=\sum_{a=1}^{N+1} \bar{\psi}_{a}^{(i)},
$$

where the subscript $a$ represent the different copies in the symmetric product. At spin $s=1$, the $\mathcal{W}_{\infty}[0]$ algebra contains 8 generators -7 are contained in the $\mathcal{N}=4$ superconformal algebra, while $V_{0}^{1}$ is the bottom component of $R^{(1)}$. Explicitly, the former are the $\mathfrak{s u}(2)_{-}$ generators

$$
\begin{aligned}
& J^{+}=-\sum_{a=1}^{N+1}: \psi_{a}^{(2)} \bar{\psi}_{a}^{(1)}:, \quad J^{-}=-\sum_{a=1}^{N+1}: \psi_{a}^{(1)} \bar{\psi}_{a}^{(2)}: \\
& J^{3}=-\frac{1}{2} \sum_{a=1}^{N+1}\left(: \psi_{a}^{(1)} \bar{\psi}_{a}^{(1)}:-: \psi_{a}^{(2)} \bar{\psi}_{a}^{(2)}:\right),
\end{aligned}
$$

\footnotetext{
${ }^{1}$ At $\lambda=0$ the $\mathfrak{s u}(2)_{+}$is only a global algebra, and the corresponding current generators only form a $\mathfrak{u}(1)^{3}$ algebra, that is described by three of the four $\mathfrak{u}(1)$ currents in (2.8) below.
} 
as well as the super-descendants of (2.6),

$$
b^{(i)}=\sum_{a=1}^{N+1} \partial \phi_{a}^{(i)}, \quad \bar{b}^{(i)}=\sum_{a=1}^{N+1} \partial \bar{\phi}_{a}^{(i)},
$$

where $i=1,2$. On the other hand, the bottom component of $R^{(1)}$ can be identified with

$$
V_{0}^{1}=-\sum_{a=1}^{N+1}\left(: \psi_{a}^{(1)} \bar{\psi}_{a}^{(1)}:+: \psi_{a}^{(2)} \bar{\psi}_{a}^{(2)}:\right) .
$$

As was mentioned above, the stringy algebra contains additional bilinear operators in the free fields that correspond to the representations $(0 ;[2,0, \ldots, 0])$ and $(0 ;[0, \ldots, 0,2])$, see [1]. The relevant wedge characters (including chemical potentials for both $\mathfrak{s u}(2)$ algebras) are

$$
\begin{aligned}
\chi_{(0 ;[2,0, \ldots, 0])}^{(\text {wedge })}=\chi_{(0 ;[0, \ldots, 0,2])}^{(\text {wedge })}=\frac{q}{(1-q)\left(1-q^{2}\right)} & \left(1+y_{1} y_{2} q^{1 / 2}\right)\left(1+y_{1} y_{2}^{-1} q^{1 / 2}\right) \\
& \times\left(1+y_{1}^{-1} y_{2} q^{1 / 2}\right)\left(1+y_{1}^{-1} y_{2}^{-1} q^{1 / 2}\right),
\end{aligned}
$$

and hence the analysis of $[1,6]$ implies that the additional generators lie in the multiplets

$$
(0 ;[2,0, \ldots, 0]): \bigoplus_{r=1}^{\infty} R^{(2 r-1)},
$$

and similarly for $(0 ;[0,0, \ldots, 0,2])$. Thus there are two additional $R^{(s)}$ multiplets for each odd spin $s$ from $(0 ;[2,0, \ldots, 0])$ and $(0 ;[0, \ldots, 0,2])$. For example, for spin $s=1$, the relevant generators are

$$
C_{2}=\sum_{a=1}^{N+1}: \psi_{a}^{(1)} \psi_{a}^{(2)}:, \quad \bar{C}_{2}=\sum_{a=1}^{N+1}: \bar{\psi}_{a}^{(1)} \bar{\psi}_{a}^{(2)}: .
$$

We have also written out explicitly the fields at $s=\frac{3}{2}$ in appendix A.

\subsection{The cubic and higher order fields}

The other fields of the stringy chiral algebra involve symmetrised higher order polynomials of the free fields. In particular, it is natural to believe that the cubic fields, i.e., those associated to the $\mathcal{W}_{\infty}[0]$ representations $(0 ;[n, 0, \ldots, 0, \bar{n}])$ with $m=n+\bar{n}=3$, lie on the first subleading Regge trajectory. Using the analysis of [6] we can read off the spin content of the relevant representations: both $(0 ;[3,0, \ldots, 0])$ and $(0 ;[0, \ldots, 0,3])$ contribute each

$$
(0 ;[3,0, \ldots, 0]) \text { and }(0 ;[0, \ldots, 0,3]): \bigoplus_{s=2}^{\infty} n(s)\left[R^{(s)}(\mathbf{2}, \mathbf{1}) \oplus R^{(s+3 / 2)}(\mathbf{1}, \mathbf{2})\right],
$$

where the multiplicities $n(s)$ are determined by the generating function (see, e.g., appendix B of [2])

$$
\frac{q^{2}}{\left(1-q^{2}\right)\left(1-q^{3}\right)}=\sum_{s=2}^{\infty} n(s) q^{s},
$$


while the $\mathcal{N}=4$ multiplets $R^{(s)}(\mathbf{2}, \mathbf{1})$ and $R^{(s)}(\mathbf{1}, \mathbf{2})$ are of the form

$$
\begin{array}{ccc} 
& s: & (\mathbf{2}, \mathbf{1}) \\
& s+\frac{1}{2}: & (\mathbf{3}, \mathbf{2}) \oplus(\mathbf{1}, \mathbf{2}) \\
R^{(s)}(\mathbf{2}, \mathbf{1}): & s+1: & (\mathbf{4}, \mathbf{1}) \oplus(\mathbf{2}, \mathbf{1}) \oplus(\mathbf{2}, \mathbf{3}) \\
& s+\frac{3}{2}: & (\mathbf{3}, \mathbf{2}) \oplus(\mathbf{1}, \mathbf{2}) \\
& s+2: & (\mathbf{2}, \mathbf{1}) \\
& s: & (\mathbf{1}, \mathbf{2}) \\
R^{(s)}(\mathbf{1}, \mathbf{2}): & s+\frac{1}{2}: & (\mathbf{2}, \mathbf{3}) \oplus(\mathbf{2}, \mathbf{1}) \\
& s+1: & (\mathbf{1}, \mathbf{4}) \oplus(\mathbf{1}, \mathbf{2}) \oplus(\mathbf{3}, \mathbf{2}) \\
& s+\frac{3}{2}: & (\mathbf{2}, \mathbf{3}) \oplus(\mathbf{2}, \mathbf{1}) \\
& s+2: & (\mathbf{1}, \mathbf{2}) .
\end{array}
$$

On the other hand, $(0 ;[2,0, \ldots, 0,1])$ and $(0 ;[1,0, \ldots, 0,2])$ contribute each

$$
(0 ;[2,0, \ldots, 0,1]) \text { and }(0 ;[1,0, \ldots, 0,2]): \quad \bigoplus_{s=3 / 2}^{\infty} m(s)\left[R^{(s)}(\mathbf{1}, \mathbf{2}) \oplus R^{(s+1 / 2)}(\mathbf{2}, \mathbf{1})\right] \text {, }
$$

where the multiplicities $m(s)$ are determined by the generating function

$$
\frac{q^{3 / 2}}{(1-q)\left(1-q^{2}\right)}=\sum_{s=3 / 2}^{\infty} m(s) q^{s}
$$

The multiplicities of the higher order families of higher spin fields can be similarly determined. For example, for $m=4$, we get from $(0 ;[2,0, \ldots, 0,2])$ the lowest order multiplets

$$
(0 ;[2,0, \ldots, 0,2]): \quad R^{(2)} \oplus R^{(5 / 2)}(\mathbf{2}, \mathbf{2}) \oplus R^{(3)} \oplus R^{(3)}(\mathbf{1}, \mathbf{3}) \oplus R^{(3)}(\mathbf{3}, \mathbf{1}) \oplus \cdots,
$$

where $R^{(s)}\left(\mathbf{d}_{1}, \mathbf{d}_{2}\right)$ is the $\mathcal{N}=4$ multiplet whose lowest component has spin $s$ and transforms in the $\left(\mathbf{d}_{1}, \mathbf{d}_{2}\right)$ under the two $\mathfrak{s u}(2)$ algebras. ${ }^{2}$ The other representations with $m=4$ start only at $s=5 / 2$ and $s=3$, respectively

$$
(0 ;[3,0, \ldots, 0,1]) \cong(0 ;[1,0, \ldots, 0,3]): \quad R^{(5 / 2)}(\mathbf{2}, \mathbf{2}) \oplus R^{(3)} \oplus R^{(3)}(\mathbf{3}, \mathbf{1}) \oplus \cdots
$$

and

$$
(0 ;[4,0, \ldots, 0]) \cong(0 ;[0, \ldots, 0,4]): \quad R^{(3)}(\mathbf{3}, \mathbf{1}) \oplus \cdots
$$

More generally, the fields that appear in the $m^{\prime}$ th column have spin $s \geq m-2$, and their number increases, for large $\operatorname{spin} s$, as $s^{m-1}$.

\section{The exactly marginal operators}

Next we need to identify the exactly marginal operators that induce the deformation of interest, i.e., that correspond to switching on the string tension. A priori, there are two

\footnotetext{
${ }^{2}$ Thus in this terminology, $R^{(s)} \equiv R^{(s)}(\mathbf{1}, \mathbf{1})$.
} 
types of exactly marginal operators, see, e.g., [15]: those that come from the untwisted sector of the symmetric orbifold, and those that arise from the 2-cycle twisted sector. The former correspond to the moduli that deform the shape and complex structure of the underlying torus, and hence should not break the higher spin symmetry; on the other hand, the 2-cycle twisted sector moduli deform the theory away from the symmetric orbifold point and will turn out to break the higher spin symmetry. A certain linear combination of these deformations describes the perturbation that switches on the string tension.

In the following, we shall construct these moduli in terms of the free fields of the symmetric orbifold; we shall then discuss the perturbation by them in turn. The exactly marginal operators that come from the untwisted sector are

$$
\begin{array}{ll}
\sum_{a=1}^{N+1}\left(\alpha_{a}^{(i)}\right)_{-1}\left(\tilde{\alpha}_{a}^{(j)}\right)_{-1}|0\rangle, & \sum_{a=1}^{N+1}\left(\bar{\alpha}_{a}^{(i)}\right)_{-1}\left(\tilde{\alpha}_{a}^{(j)}\right)_{-1}|0\rangle, \\
\sum_{a=1}^{N+1}\left(\alpha_{a}^{(i)}\right)_{-1}\left(\tilde{\bar{\alpha}}_{a}^{(j)}\right)_{-1}|0\rangle, & \sum_{a=1}^{N+1}\left(\bar{\alpha}_{a}^{(i)}\right)_{-1}\left(\tilde{\bar{\alpha}}_{a}^{(j)}\right)_{-1}|0\rangle,
\end{array}
$$

where $\left(\alpha_{a}^{(i)}\right)_{n}$ and $\left(\bar{\alpha}_{a}^{(i)}\right)_{n}$ are the modes associated to $\partial \phi_{a}^{(i)}$ and $\partial \bar{\phi}_{a}^{(i)}$, respectively. We shall confirm below, see section 4 , that these deformations do not break the $\mathcal{W}_{\infty}[0]$ algebra.

\subsection{Exactly marginal operators in the twisted sector}

The other exactly marginal operators arise from the 2-cycle twisted sector, i.e., they are the half-descendants of the BPS states with $h=\bar{h}=\frac{1}{2}$. From the coset viewpoint, the ground state of the relevant twisted sector transforms in the representation [1]

$$
\left(\left[\frac{k}{2}, 0,0, \ldots, 0\right] ;\left[\frac{k}{2}+1,0,0, \ldots, 0\right] ; k+(N+2)\right) .
$$

However, for the purpose of doing the actual perturbation calculation, it is more convenient to describe them directly in terms of the symmetric orbifold language.

For definiteness let us consider the 2-cycle twisted sector corresponding to the permutation (12). Then the free fields with $a \geq 3$ behave as before, while out of the two fields associated to $a=1,2$, we can form the antisymmetric and symmetric combination

$$
P^{(i) \mathrm{A}}=\frac{1}{\sqrt{2}}\left(P_{1}^{(i)}-P_{2}^{(i)}\right), \quad P^{(i) \mathrm{S}}=\frac{1}{\sqrt{2}}\left(P_{1}^{(i)}+P_{2}^{(i)}\right),
$$

where $P$ stands for one of $\psi, \bar{\psi}, \partial \phi$ or $\partial \bar{\phi}$. The $P^{(i) \mathrm{S}}$ fields have modes with the usual mode numbers (integers for bosons, half-integer for fermions in the NS-sector), while for the $P^{(i) \mathrm{A}}$ fields, the moding is reversed, i.e., integer for the fermions in the NS-sector, and half-integer for the bosons. The non-trivial OPEs between these combinations are (for the case of the left-moving fermions)

$$
\begin{aligned}
\psi^{(i) \mathrm{A}}(z) \bar{\psi}^{(j) \mathrm{A}}(w) & \sim \frac{\delta^{i j}}{z-w}+\mathcal{O}(1) \\
\psi^{(i) \mathrm{S}}(z) \bar{\psi}^{(j) \mathrm{S}}(w) & \sim \frac{\delta^{i j}}{z-w}+\mathcal{O}(1) .
\end{aligned}
$$


Note that under the (12) permutation action, the symmetric combination is invariant, while the anti-symmetric combination picks up a sign. Thus the states that survive the orbifold projection involve an even number of odd generators (both for left- and right-movers). This condition will then also guarantee that the surviving states satisfy $h-\bar{h} \in \mathbb{Z}$.

\subsubsection{The perturbing fields}

Next we need to introduce some notation for the twisted sector ground states. Let us denote the ground state of the twisted sector that is characterised by

$$
\psi_{0}^{(1) \mathrm{A}}\left|\Psi^{0}\right\rangle=0, \quad \psi_{0}^{(2) \mathrm{A}}\left|\Psi^{0}\right\rangle=0
$$

as $\left|\Psi^{0}\right\rangle$. Then by applying the fermionic zero modes we define the states

$$
\left|\Psi^{+}\right\rangle=\bar{\psi}_{0}^{(1) \mathrm{A}}\left|\Psi^{0}\right\rangle, \quad\left|\Psi^{-}\right\rangle=\bar{\psi}_{0}^{(2) \mathrm{A}}\left|\Psi^{0}\right\rangle, \quad\left|\Psi^{3}\right\rangle=\bar{\psi}_{0}^{(1) \mathrm{A}} \bar{\psi}_{0}^{(2) \mathrm{A}}\left|\Psi^{0}\right\rangle
$$

The $\left|\Psi^{ \pm}\right\rangle$transform as a doublet under the $\mathfrak{s u}(2)$ - algebra, while the $\left|\Psi^{0}\right\rangle$ and $\left|\Psi^{3}\right\rangle$ are singlets. The actual perturbing states are then the super-descendants of the BPS gound states $\left|\Psi^{ \pm}\right\rangle$. There are only two non-vanishing, independent descendants, which we denote as

$$
\begin{aligned}
\left|\Phi^{+}\right\rangle & =\left(-\alpha_{-\frac{1}{2}}^{(1) \mathrm{A}} \bar{\psi}_{0}^{(1) \mathrm{A}} \bar{\psi}_{0}^{(2) \mathrm{A}}+\bar{\alpha}_{-\frac{1}{2}}^{(2) \mathrm{A}}\right)\left|\Psi^{0}\right\rangle \\
\left|\Phi^{-}\right\rangle & =\left(\bar{\alpha}_{-\frac{1}{2}}^{(1) \mathrm{A}}+\alpha_{-\frac{1}{2}}^{(2) \mathrm{A}} \bar{\psi}_{0}^{(1) \mathrm{A}} \bar{\psi}_{0}^{(2) \mathrm{A}}\right)\left|\Psi^{0}\right\rangle .
\end{aligned}
$$

With the above normalisation conventions, these states have unit norm and are orthogonal to one another. The \pm label can be identified with the charge under the global $\mathfrak{s u}(2)$ algebra. Obviously, there are similar anti-chiral states (that have to be combined with these), and in total there are therefore 4 exactly marginal deformations. However, only one combination of the 4 deformations preserves the global $\mathrm{SO}(4)$ symmetry; this is the exactly marginal operator that corresponds to switching on the string tension. For the computations in this paper, the precise form of this combination is, however, not important since the rightmoving perturbing field only enters rather trivially, and the effect of the perturbation seems to be independent of which of the two left-moving super-descendants $\left|\Phi^{ \pm}\right\rangle$of the ground state are considered. We have done most of the following computations for the case of $\left|\Phi^{+}\right\rangle$; we have also checked in some cases (in particular for the quadratic operators up to spin $s \leq 5)$ that the perturbation by $\left|\Phi^{-}\right\rangle$leads exactly to the same conclusion.

\section{First order deformation analysis}

With these preparations we can now study the behaviour of the chiral fields under the perturbation by the exactly marginal field $|\Phi\rangle$ from above. (As was mentioned there, we have mainly done the analysis for $|\Phi\rangle \equiv\left|\Phi^{+}\right\rangle$.) To first order, i.e., considering the 3-point function that involves two chiral fields and one perturbing field, the answer is always trivial. This is simply a consequence of the fact that the perturbing field has $h=\bar{h}=1$, and since the other two fields have $\bar{h}=0$, the anti-chiral part of the correlation function vanishes, see also the discussion in [16]. 
Nevertheless, there is a 'first-order' analysis that determines whether a given chiral field will pick up an anomalous dimension. The criterion for the spin $s$ field $W^{(s)}$ of the chiral algebra to remain chiral is that, see e.g., [16]

$$
\mathcal{N}\left(W^{(s)}\right) \equiv \sum_{l=0}^{\left\lfloor s+h_{\Phi}\right\rfloor-1} \frac{(-1)^{l}}{l !}\left(L_{-1}\right)^{l} W_{-s+1+l}^{(s)} \Phi=0,
$$

where $\Phi$ is the perturbing state. Note that we have not assumed that $\Phi$ is primary, and hence the sum in (4.1) runs over a slightly larger index set than in [16]. Actually, $\mathcal{N}\left(W^{(s)}\right)$ has the interpretation $[17,18]$

$$
\partial_{\bar{z}} W^{(s)}(z, \bar{z})=g \pi \mathcal{N}\left(W^{(s)}\right)
$$

where $g$ is the coupling constant.

\subsection{Deformation by the untwisted sector perturbation fields}

Let us begin by studying the perturbation by the untwisted sector fields (3.1). The chiral fields at $s=\frac{1}{2}$, see eq. (2.6), are purely fermionic and are not affected by the bosonic perturbations (3.1). The same statement applies to all the spin $s=1$ fields, except those described by eq. (2.8). For the latter, the corresponding zero modes vanish on $\Phi$, and the +1 modes map $\Phi$ to the vacuum, which in turn is annihilated by $L_{-1}$. Hence, we conclude that $\mathcal{N}=0$ in (4.1), and thus also all spin $s=1$ fields are not lifted by this perturbation. We should mention that a similar conclusion was also reached in [19], where the perturbation by the field $(\mathrm{f} ; \overline{\mathrm{f}})$ was studied. For generic $k$ this perturbation breaks the spin $s=1$ symmetry, see eq. (5.4) of [19], but the effect disappears in the $k \rightarrow \infty$ limit (in which the $(f ; \bar{f})$ field is closely related to the above deformations).

We can carry out a similar analysis for the generators of higher spin, and we find that, as expected, the perturbation by the untwisted sector fields (3.1) does not break any of the $\mathcal{W}_{\infty}[0]$ generators, nor those of the stringy extension.

\subsection{Deformation by the twisted sector perturbation fields}

For the case of the perturbation by a twisted sector field, the situation is more interesting. By construction, the perturbing fields (3.8) and (3.9) are $\mathfrak{s u}(2)_{-}$singlets,

$$
J_{0}^{\alpha}\left|\Phi^{ \pm}\right\rangle=0 \quad \alpha \in\{3, \pm\}
$$

Thus it follows that the perturbation by $\Phi=\Phi^{ \pm}$preserves the $\mathfrak{s u}(2)_{-}$symmetry. On the other hand, for the spin 1 current from the first non-trivial $\mathcal{N}=4$ multiplet, we find

$$
\begin{aligned}
\mathcal{N}\left(V^{(1) 0}\right) & =\left(V^{(1) 0}\right)_{0}\left|\Phi^{+}\right\rangle \\
& =\left(\bar{\psi}_{0}^{(1) \mathrm{A}} \psi_{0}^{(1) \mathrm{A}}+\bar{\psi}_{0}^{(2) \mathrm{A}} \psi_{0}^{(2) \mathrm{A}}-1\right)\left(-\alpha_{-\frac{1}{2}}^{(1) \mathrm{A}} \bar{\psi}_{0}^{(1) \mathrm{A}} \bar{\psi}_{0}^{(2) \mathrm{A}}+\bar{\alpha}_{-\frac{1}{2}}^{(2) \mathrm{A}}\right)\left|\Psi^{0}\right\rangle \\
& =\left(-\alpha_{-\frac{1}{2}}^{(1) \mathrm{A}} \bar{\psi}_{0}^{(1) \mathrm{A}} \bar{\psi}_{0}^{(2) \mathrm{A}}-\bar{\alpha}_{-\frac{1}{2}}^{(2) \mathrm{A}}\right)\left|\Psi^{0}\right\rangle
\end{aligned}
$$


The resulting state does not vanish, and hence the spin 1 field $V^{(1) 0}$ is not conserved by the perturbation. A similar computation can also be done for the other generators of the extended chiral algebra with a similar result. Thus it appears that the perturbation by $\Phi^{+}$ breaks the higher spin symmetry down to the $\mathcal{N}=4$ superconformal algebra. This is what one should expect for the perturbation by the field that switches on the tension. A similar conclusion applies also to $\Phi^{-}$.

\section{$5 \quad$ Second order deformation analysis}

The analysis of the previous section has given some evidence for the fact that the perturbation by $\Phi=\Phi^{ \pm}$breaks the higher spin symmetry down to the $\mathcal{N}=4$ superconformal algebra. In this section we want to calculate the relevant anomalous dimensions quantitatively; this will allow us to determine the masses of the corresponding fields, and hence enable us to see whether these fields lie on different Regge trajectories. In order to do this computation, we now need to perform a second order analysis.

Let us begin by explaining the general structure of our computation. We consider the normalised perturbed two point functions

$$
\left\langle W^{(s) i}\left(z_{1}\right) W^{(s) j}\left(z_{2}\right)\right\rangle_{\Phi}=\frac{\left\langle W^{(s) i}\left(z_{1}\right) W^{(s) j}\left(z_{2}\right) e^{\Delta S}\right\rangle}{\left\langle e^{\Delta S}\right\rangle}, \quad \Delta S=g \int d^{2} w \Phi(w, \bar{w}),
$$

where the coupling constant $g$ is dimensionless. Expanding in powers of $g$, we get

$$
\begin{aligned}
& \left\langle W^{(s) i}\left(z_{1}\right) W^{(s) j}\left(z_{2}\right)\right\rangle_{\Phi}-\left\langle W^{(s) i}\left(z_{1}\right) W^{(s) j}\left(z_{2}\right)\right\rangle \\
& =\frac{g^{2}}{2}\left(\int d^{2} w_{1} d^{2} w_{2}\left\langle W^{(s) i}\left(z_{1}\right) W^{(s) j}\left(z_{1}\right) \Phi\left(w_{1}, \bar{w}_{1}\right) \Phi\left(w_{2}, \bar{w}_{2}\right)\right\rangle\right. \\
& \left.\quad-\int d^{2} w_{1} d^{2} w_{2}\left\langle W^{(s) i}\left(z_{1}\right) W^{(s) j}\left(z_{2}\right)\right\rangle\left\langle\Phi\left(w_{1}, \bar{w}_{1}\right) \Phi\left(w_{2}, \bar{w}_{2}\right)\right\rangle\right)+\mathcal{O}\left(g^{3}\right),
\end{aligned}
$$

where the $\mathcal{O}(g)$ term vanishes as explained at the beginning of the previous section, and hence the leading correction to the 2-pt function appears at second order. To read off the anomalous dimension from this calculation we note that the 2-point function of any quasiprimary operator is of the form

$$
\left\langle W^{(s) i}\left(z_{1}\right) W^{(s) j}\left(z_{1}\right)\right\rangle_{\Phi} \sim \frac{c^{i j}}{\left(z_{1}-z_{2}\right)^{2\left(s+\gamma^{i j}\right)}\left(\bar{z}_{1}-\bar{z}_{2}\right)^{2 \bar{\gamma}^{i j}}},
$$

which can be expanded, for small $\gamma^{i j}$, as

$$
\left\langle W^{(s) i}\left(z_{1}\right) W^{(s) j}\left(z_{1}\right)\right\rangle_{\Phi} \sim \frac{c^{i j}}{\left(z_{1}-z_{2}\right)^{2 s}}\left(1-2 \gamma^{i j} \ln \left(z_{1}-z_{2}\right)-2 \bar{\gamma}^{i j} \ln \left(\bar{z}_{1}-\bar{z}_{2}\right)+\cdots\right),
$$

where $\gamma^{i j}=\bar{\gamma}^{i j}$, because of locality. Thus we can read off the anomalous dimension $\gamma^{i j}$ from the log-term in the perturbed 2-point function. In general, however, we need to 'diagonalise' the perturbed 2-point functions as typically different fields (of the same unperturbed conformal dimension) will mix at this order. In order to simplify the analysis 
we shall assume that the fields $W^{(s) i}$ for $i=1, \ldots, N(s)$ form an orthonormal basis of the spin $s$ fields in the unperturbed theory, i.e., that

$$
\left\langle W^{(s) i}\left(z_{1}\right) W^{(s) j}\left(z_{2}\right)\right\rangle=\delta^{i j}\left(z_{1}-z_{2}\right)^{-2 h_{i}} .
$$

Then the relevant mixing matrix that we have to diagonalise turns out to equal (see appendix B, where we present two different approaches to this calculation)

$$
\gamma^{i j}=\frac{g^{2} \pi^{2}\left\langle\left[\mathcal{N}\left(W^{(s) i}\right)\right]\left(z_{1}\right)\left[\left(\mathcal{N}\left(W^{(s) j}\right)\right]\left(z_{2}\right)\right\rangle\right.}{\left(z_{1}-z_{2}\right)^{h_{i}+h_{j}}}
$$

where $\mathcal{N}(W)$ was defined in (4.1). Writing out the definition of $\mathcal{N}(W)$, this can be further simplified to

$$
\gamma^{i j}=g^{2} \pi^{2} \sum_{m=1-s}^{s \bmod 1}(-1)^{\lceil s\rceil-1-\lfloor m\rfloor}\left(\begin{array}{c}
2 s-2 \\
s-1-m
\end{array}\right)\left\langle\Phi\left|W_{-m}^{(s) i} W_{m}^{(s) j}\right| \Phi\right\rangle .
$$

One can also arrive at the same conclusion following the analysis in [20]. To this end we observe that, because of locality, the anomalous dimension must be the same for the leftand right-moving conformal dimension. Then, since $\bar{L}_{-1}=\partial_{\bar{z}}$, the anomalous dimension matrix is proportional to

$$
\left\langle\partial_{\bar{z}} W^{(s) i} \mid \partial_{\bar{z}} W^{(s) j}\right\rangle=2\left\langle W^{(s) i}\left|\bar{L}_{0}\right| W^{(s) j}\right\rangle,
$$

where we have used that $\bar{L}_{-1}^{\dagger}=\bar{L}_{1}$, as well as the fact that $\bar{L}_{1} W^{(s) j}=0$. On the other hand, using (4.2), the left-hand-side can be described in terms of the $\mathcal{N}\left(W^{(s) i}\right)$, and thus the matrix elements of $\bar{L}_{0}$ in the orthonormal basis $W^{(s) k}$ equal the matrix elements of $\mathcal{N}\left(W^{(s) k}\right) \cdot^{3}$

\subsection{A more structural approach}

We can also understand some of the entries of the mixing matrix using the $\mathcal{W}$-algebra representation theory. In order to explain this, let us consider the case where $W^{(s)}$ and $W^{(t)}$ are $\mathcal{W}$-highest weight states of spin $s$, and $t$, respectively. Then a certain $W^{(s)}$ descendant' of $W^{(t)}$

$$
W^{s+t-1}=\sum_{m=0} \frac{(-1)^{m}(2 s-2)_{(m)}}{m !(2 s+2 t-4)_{(m)}}\left(L_{-1}\right)^{m} W_{m+1-s}^{(s)} W^{(t)} \equiv \mathcal{N}\left(W^{(s)}\right)_{t} W^{(t)}
$$

defines a quasiprimary field of spin $s+t-1$. For the calculation of the mixing matrix, we need to determine the associated vector of eq. (4.1), i.e.,

$$
\begin{aligned}
\mathcal{N}\left(W^{s+t-1}\right)= & \sum_{p^{\prime}=0}^{s+t-1} \frac{(-1)^{p^{\prime}}}{p^{\prime} !}\left(L_{-1}\right)^{p^{\prime}} W_{-(s+t-1)+1+p^{\prime}}^{s+t-1} \Phi \\
= & \frac{1}{\left(\begin{array}{c}
2 s+2 t-4 \\
2 s-2
\end{array}\right)} \sum_{p^{\prime}=0}^{s+t-1} \sum_{k=0}^{2 s-2}\left(\begin{array}{c}
p^{\prime} \\
k
\end{array}\right)\left(\begin{array}{c}
2 s+2 t-4-p^{\prime} \\
2 s-2-k
\end{array}\right) \frac{(-1)^{p^{\prime}}}{p^{\prime} !} \\
& \times\left(L_{-1}\right)^{p^{\prime}}\left[W_{1-s+k}^{(s)}, W_{-t+1+p^{\prime}-k}^{(t)}\right] \Phi .
\end{aligned}
$$

\footnotetext{
${ }^{3}$ We thank Wei Li for a discussion about this point.
} 
After a slightly tedious computation, we find that the resulting vector can be written as

$$
\mathcal{N}\left(W^{s+t-1}\right)=\mathcal{N}\left(W^{(s)}\right)_{t} \mathcal{N}\left(W^{(t)}\right)_{1} \Phi-\mathcal{N}\left(W^{(t)}\right)_{s} \mathcal{N}\left(W^{(s)}\right)_{1} \Phi
$$

where $\mathcal{N}\left(W^{(s)}\right)_{t}$ was already implicitly defined in (5.9), and is explicitly given as

$$
\mathcal{N}\left(W^{(s)}\right)_{t}=\sum_{m=0} \frac{(-1)^{m}(2 s-2)_{(m)}}{m !(2 s+2 t-4)_{(m)}}\left(L_{-1}\right)^{m} W_{m+1-s}^{(s)} .
$$

(Note that $\mathcal{N}\left(W^{(s)}\right)_{1} \Phi=\mathcal{N}\left(W^{(s)}\right)$, see eq. (4.1).) This formula leads to constraints among the entries of the mixing matrix; for example, it shows directly that if both $W^{(s)}$ and $W^{(t)}$ do not acquire an anomalous dimension, i.e., $\mathcal{N}\left(W^{(s)}\right)=\mathcal{N}\left(W^{(t)}\right)=0$, the same is true for the $W^{(s)}$-descendant $W^{s+t-1}$ of $W^{(t)}$.

\subsection{Relation to bulk masses}

Ultimately, we are not directly interested in the anomalous dimensions of the higher spin fields, but in the associated bulk masses. It is well known that the bulk mass of an arbitrary $p$-form field is related to the conformal dimension on the boundary via, see, e.g., [21]

$$
\Delta=\frac{1}{2}\left(2+2 \sqrt{(1-p)^{2}+m^{2}}\right) .
$$

Solving for the mass $m$ leads to the equation

$$
m^{2}=(\Delta-1)^{2}-(p-1)^{2}
$$

In particular, if $\Delta=p$, then $m^{2}=0$. Now, if the spin $s$ operator with $(h, \bar{h})=(s, 0)$ and $\Delta=h+\bar{h}=s$ acquires an anomalous dimension, its bulk mass becomes

$$
\begin{aligned}
m^{2} & =(s+\gamma(s)-1)^{2}-(s-1)^{2} \\
& =\gamma(s)(2 s+\gamma(s)-2) \approx 2 \gamma(s)(s-1),
\end{aligned}
$$

where we have used the approximation that the anomalous dimension is much smaller than the spin. A standard flat space Regge trajectory would therefore correspond to the situation where the anomalous dimension $\gamma(s)$ is a constant, independent of $s$. As we will see below, it seems that for the case at hand, the anomalous dimension behaves as $\gamma(s) \sim \log s$, at least without taking the mixing between the different fields into account.

\section{Explicit anomalous dimensions}

Now we have accumulated all the ingredients to derive explicit expressions for the anomalous dimensions. Before we get started with describing the explicit results, there are a few general features of the results we should point out. 


\subsection{The structure of the analysis}

In general, the matrix $\gamma^{i j}$ will not be diagonal, and in order to extract the anomalous dimensions from the calculation of (5.7), we need to diagonalise it. As we have reviewed in section 2, the spin fields can be organised in columns labeled by the number of the underlying free fields $m=2,3, \ldots$, see eq. (2.3). The fields that appear in the $m$ 'th column have spin $s \geq m-2$, and since to leading order only fields of the same conformal dimension have non-trivial matrix elements $\gamma^{i j}$, for any fixed spin, the spin $s$ part of the matrix $\gamma^{i j}$ is finite-dimensional.

There are some selection rules that guarantee that not all the different spin fields can mix with one another. First of all, since all the free fields carry non-trivial $\mathfrak{u}(1)$ charge, it is easy to see that mixing can only take place between fields from the $\mathcal{W}_{\infty}$ representations $(0 ;[n, 0, \ldots, 0, \bar{n}])$ for which $n-\bar{n}$ has the same value. As a consequence, only columns of order $m$ (with $m=n+\bar{n}$ ) that differ by an even number, can mix. Furthermore, since the perturbation does not break the $\mathcal{N}=4$ superconformal algebra, the anomalous dimensions must be the same for each member of a given $\mathcal{N}=4$ multiplet - we have also checked this explicitly for a few cases - and indeed only fields that sit in the same $\mathcal{N}=4$ multiplets can mix. Because of this, we shall always give the anomalous dimension of full $\mathcal{N}=4$ multiplets, and we shall label them by the spin of the lowest field (and the appropriate $\mathfrak{s u}(2)_{+} \oplus \mathfrak{s u}(2)_{-}$labels $)$.

However, even taking both of these considerations into account, the problem of diagonalising the complete mixing matrix becomes quickly very complicated, and we have only solved it completely for rather low spin, see section 6.2 below. For higher values of $s$, we have only performed the diagonalisation within the subset of bilinear and trilinear fields, see section 6.3 .

\subsection{The anomalous dimensions at low spin}

In this section we give our explicit results for small spin. Let us consider the different values in turn.

\subsubsection{Spin 1}

At spin 1 we have the lowest multiplet of the original higher spin algebra, i.e., $R^{(1)}$ from (2.4). In addition, we have a conjugate pair of $R^{(1)}$ representations coming from (2.11). Because of $\mathfrak{u}(1)$ charge conservation, these fields cannot mix, and hence the relevant $\gamma^{i j}$ matrix is already automatically diagonal. All its diagonal entries turn out to equal

$$
\delta h\left(R^{(1)}\right)_{m=2}=\frac{1}{2} \frac{g^{2} \pi^{2}}{N+1} .
$$

\subsubsection{Spin $\frac{3}{2}$}

At spin $\frac{3}{2}$, the only muliplets arise from the $(0 ;[2,0, \ldots, 0,1])$ and $(0 ;[1,0, \ldots, 0,2])$ sectors, see eq. (2.17). Since these two multiplets cannot mix (because they have different $\mathfrak{u}(1)$ charge), the $\gamma^{i j}$ matrix is again diagonal, and we can read off the anomalous dimensions 
from the diagonal entries. They turn out to equal

$$
\delta h\left(R^{(3 / 2)}\right)_{m=3}=\frac{3}{4} \frac{g^{2} \pi^{2}}{N+1} .
$$

\subsubsection{Spin 2}

The situation at spin 2 is more interesting. There is one $R^{(2)}$ multiplet from the original higher spin algebra, see eq. (2.4). It can mix with the $R^{(2)}$ multiplet from the $m=4$ term in the $(0 ;[2,0, \ldots, 0,2])$ representation, see eq. $(2.19)$. The mixing matrix turns out to be

$$
\gamma^{i j}\left(R^{(2)}\right)=\frac{g^{2} \pi^{2}}{N+1}\left(\begin{array}{cc}
\frac{9}{16} & \frac{3}{16} \sqrt{6} \\
\frac{3}{16} \sqrt{6} & \frac{15}{8}
\end{array}\right),
$$

and, after diagonalisation, it leads to the two anomalous dimensions

$$
\delta h\left(R^{(2)}\right)_{m=2}=0.418 \frac{g^{2} \pi^{2}}{N+1}, \quad \delta h\left(R^{(2)}\right)_{m=4}=2.020 \frac{g^{2} \pi^{2}}{N+1},
$$

where we have made the assignment $m=2$ and $m=4$ since the relevant eigenvectors are predominantly from the $m=2$ and $m=4$ part, respectively. (The relevant prefactors are relatively close to $\frac{9}{16}=0.562$ and $\frac{15}{8}=1.875$.)

In addition, there is one pair of $R^{(2)}(\mathbf{2}, \mathbf{1})$ multiplets in the $(0 ;[3,0, \ldots, 0])$ and $(0 ;[0, \ldots, 0,3])$ representations, see eq. (2.13). Again these states cannot mix since they have opposite $\mathfrak{u}(1)$ charge, and the anomalous dimensions turn out to equal

$$
\delta h\left(R^{(2)}(\mathbf{2}, \mathbf{1})\right)_{m=3}=\frac{3}{2} \frac{g^{2} \pi^{2}}{N+1} .
$$

Similarly, a pair of $R^{(2)}(\mathbf{2}, \mathbf{1})$ multiplets sit in the $(0 ;[1,0, \ldots, 0,2])$ representation and its conjugate, $(0 ;[2,0, \ldots, 0,1])$, see eq. (2.17), and their anomalous dimensions also turn out to equal

$$
\delta h\left(R^{(2)}(\mathbf{2}, \mathbf{1})\right)_{m=3}=\frac{3}{2} \frac{g^{2} \pi^{2}}{N+1} .
$$

\subsubsection{Spin $\frac{5}{2}$}

At higher spin we have only evaluated the anomalous dimensions for some states. For example, at spin $s=5 / 2$, the multiplet $R^{(5 / 2)}(\mathbf{2}, \mathbf{2})$ from the $(0 ;[2,0, \ldots, 0,2])$ representation at $m=4$ does not participate in mixing with other multiplets, and its anomalous dimension equals

$$
\delta h\left(R^{(5 / 2)}(\mathbf{2}, \mathbf{2})\right)=\frac{5}{2} \frac{g^{2} \pi^{2}}{N+1}
$$

\subsubsection{Spin 3}

We have also worked out some examples at spin 3. First, of all there is mixing between the $R^{(3)}$ multiplet of the original higher spin algebra and the $R^{(3)}$ multiplet in the $(0 ;[2,0, \ldots, 0,2])$ representation at $m=4$. The mixing matrix is of the form

$$
\gamma^{i j}\left(R^{(3)}\right)=\frac{g^{2} \pi^{2}}{N+1}\left(\begin{array}{cc}
\frac{21}{32} & \frac{55 \sqrt{5}}{512 \sqrt{2}} \\
\frac{55 \sqrt{5}}{512 \sqrt{2}} & \frac{2435}{768}
\end{array}\right),
$$


and the anomalous dimensions are, upon the diagonalisation of the mixing matrix,

$$
\delta h\left(R^{(3)}\right)_{m=2}=0.645 \frac{g^{2} \pi^{2}}{N+1}, \quad \delta h\left(R^{(3)}\right)_{m=4}=3.182 \frac{g^{2} \pi^{2}}{N+1} .
$$

There is furthermore one $R^{(3)}(\mathbf{2}, \mathbf{1})$ multiplet at $m=3$ term in the $(0 ;[2,0, \ldots, 0,1])$ representation, see eq. (2.17). It can mix with the $R^{(3)}(\mathbf{2}, \mathbf{1})$ multiplet from the $(0 ;[3,0, \ldots, 0,2])$ representation at $m=5$. The mixing matrix turns out to be

$$
\gamma^{i j}\left(R^{(3)}(\mathbf{2}, \mathbf{1})\right)=\frac{g^{2} \pi^{2}}{N+1}\left(\begin{array}{cc}
\frac{51}{32} & \frac{9}{8} \sqrt{2} \\
\frac{9}{8} \sqrt{2} & \frac{21}{4}
\end{array}\right),
$$

and, after diagonalisation, it leads to the two anomalous dimensions

$$
\delta h\left(R^{(3)}(\mathbf{2}, \mathbf{1})\right)_{m=3}=0.998 \frac{g^{2} \pi^{2}}{N+1}, \quad \delta h\left(R^{(3)}(\mathbf{2}, \mathbf{1})\right)_{m=5}=5.845 \frac{g^{2} \pi^{2}}{N+1}
$$

where we have made the assignment as in the previous case. There is also a multiplet $R^{(3)}(\mathbf{3}, \mathbf{1})$ coming from the $(0 ;[2,0, \ldots, 0,2])$ representation at $m=4$, see eq. $(2.19)$; it cannot mix with any other multiplet, and its anomalous dimension therefore equals the diagonal entry which is

$$
\delta h\left(R^{(3)}(\mathbf{3}, \mathbf{1})\right)_{m=4}=\frac{15}{4} \frac{g^{2} \pi^{2}}{N+1} .
$$

\subsection{Partial diagonalisation for higher spin}

Unfortunately, the size of the mixing matrix increases rather quickly with the spin, and the calculation becomes soon very complicated. In particular, it is therefore not feasible to determine the large spin behaviour of the anomalous dimensions in this manner. In order to obtain some idea of the functional form, we have resorted to studying the partial diagonalisation problem where we only diagonalise the mixing matrix among the fields of a given value of $m$. In particular, for the 'leading' $m=2$ family this is rather simple since no mixing can take place: the original higher spin fields (2.4) and the fields from (2.11) cannot mix among each other since they have different $\mathfrak{u}(1)$ charge, and in each family, there is only one multiplet of a given spin. Thus this $\gamma^{i j}$ submatrix is automatically diagonal, and it is enough to determine the diagonal matrix elements. This can be done in closed form with the result

$$
\gamma^{(s)}=\frac{g^{2} \pi^{2} \sum_{p=0}^{s}(-1)^{s-p}\left(\begin{array}{c}
2 s \\
s-p
\end{array}\right) P_{2}(s, p)}{(N+1) E_{2}(s)}
$$


where

$$
\begin{aligned}
E_{2}(s)= & \sum_{q=0}^{s-1} \sum_{p=0}^{s-1}(-1)^{s+1+p+q}\left(\begin{array}{l}
s \\
q
\end{array}\right)\left(\begin{array}{c}
s \\
q+1
\end{array}\right)\left(\begin{array}{l}
s \\
p
\end{array}\right)\left(\begin{array}{c}
s \\
p+1
\end{array}\right) \\
& \times\left((-2)_{(q)}(-2-q)_{(s-p-1)}(-2)_{(s-q-1)}(q-s-1)_{(p)}\right), \\
P_{2}(s, p)= & \sum_{n=3 / 2}^{p-3 / 2} n(p-n) f(s, p, n) f(s,-p, n-p) \\
& +\frac{3}{2}(-1)^{s+1} \Theta(p-2) f(s, p, 1 / 2) f(s,-p,-1 / 2)(p-1 / 2) \\
& +\frac{1}{2} \delta_{p, 1} f(s, 1,1 / 2) f(s,-1,-1 / 2), \\
& s-1 \\
f(s, p, n)= & \sum_{q=0}(-1)^{q}\left(\begin{array}{c}
s \\
q
\end{array}\right)\left(\begin{array}{c}
s \\
q+1
\end{array}\right)(-1-p+n)_{(s-q-1)}(-1-n)_{(q)},
\end{aligned}
$$

and $s$ is the spin of the higher spin current multiplet. Here $(a)_{(n)}$ is defined by

$$
(a)_{(n)}=a(a-1) \cdots(a-n+1) .
$$

This formula was derived for the multiplets of spin $s$ arising from (2.4); for the multiplets from (2.11), the relevant formula looks different (see the ancillary file), but gives rise to exactly the same values for $\gamma^{(s)}$ (for odd spin $s$ ). For the multiplets up to $s \leq 5$, we have worked out the anomalous dimensions separately for each member of the multiplet (and confirmed that it is indeed the same for all the fields of a given multiplet); for $s \geq 6$, the analysis was done for a specific state in the $(\mathbf{3}, \mathbf{1})$ representation at $h=s+1$.

While we have not been able to simplify the formula for $\gamma^{(s)}$ for general $s$, we have plotted the result, see the solid blue curve in the log-log plot of figure 1 . The resulting curve can be fitted quite well by the function

$$
\gamma_{2}^{(s)}=0.20293 \log (7.04703 s+3.84921) .
$$

We have also done a similar analysis for the fields with $m=3$. While the fields from the different sectors $(0 ;[n, 0, \ldots, 0, \bar{n}])$ with $n+\bar{n}=3$ cannot mix (because of $\mathfrak{u}(1)$ charge conservation), non-trivial mixing will occur due to the multiplicities $n(s)>1$ and $m(s)>1$ in eqs. (2.14) and (2.18), respectively. We have solved this mixing problem explicitly up to spin $s \leq 14$, and we have plotted these partially diagonalised eigenvalues by the brown dots in figure 1. For larger spin, we have again only worked out the diagonal matrix elements for one family of fields that transforms in the $(\mathbf{4}, \mathbf{1})$ representation; the curve that fits these diagonal anomalous dimensions is

$$
\gamma_{3}^{(s)}=0.476933 \log (7.61932 s+14.7676),
$$

and it is also included (as the dashed brown curve) in the log-log plot of figure 1. In this figure we have also included the exact eigenvalues of some low-lying fields (involving up to $m=5$ ), some of which were explicitly described in section 6.2 ; in those cases, where there was a genuine mixing, we have also indicated with an arrow the shift in eigenvalue due to the mixing. 


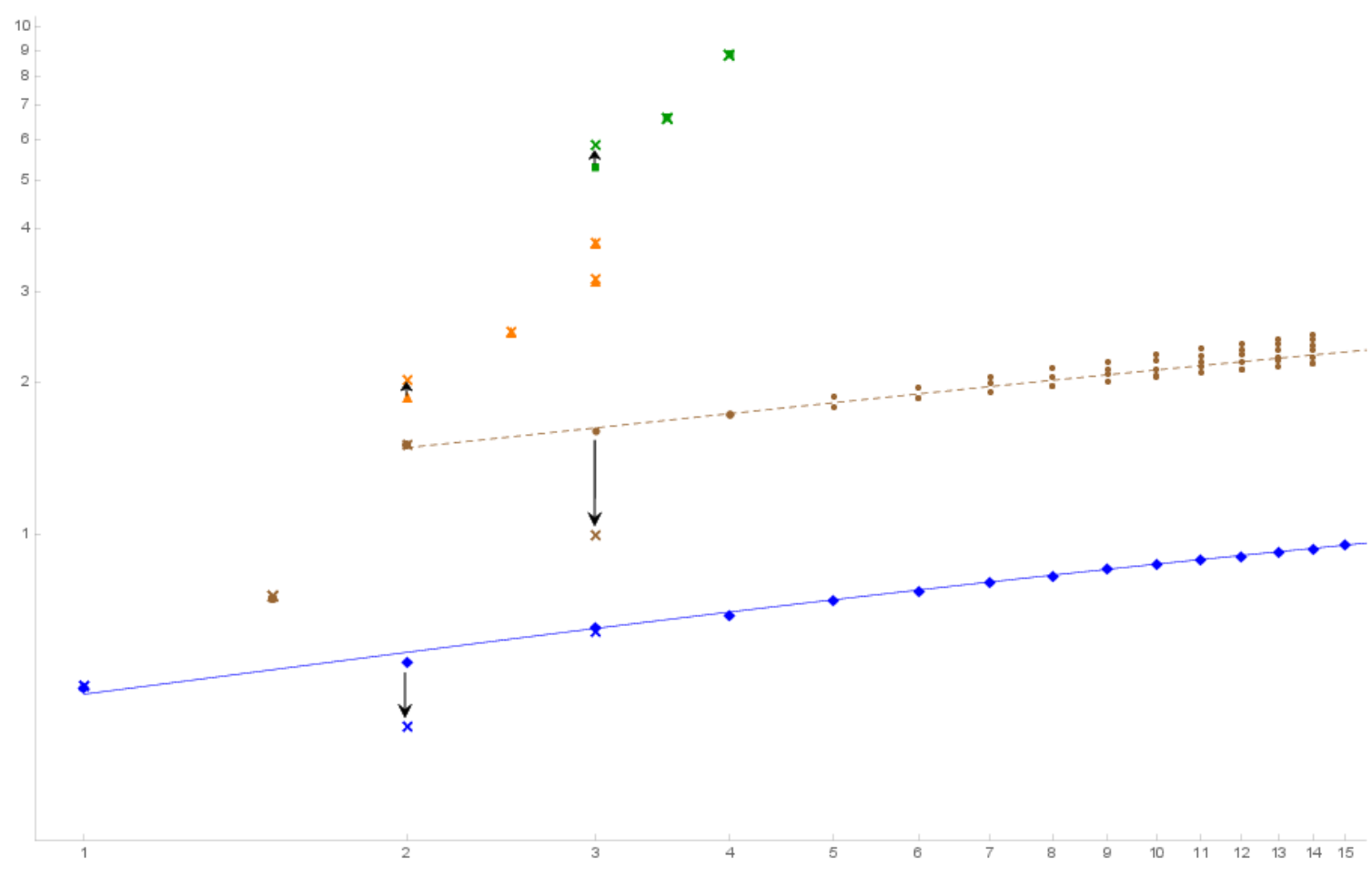

Figure 1. The anomalous dimensions, in units of $\pi^{2} g^{2} /(N+1)$, as a function of the spin in a $\log$-log plot. Dots represent the diagonal values (e.g., for $m=2$ ), or the eigenvalues after partial diagonalisation among the fields of the corresponding value of $m$ (e.g., for $m=3$ ), while crosses describe the actual eigenvalues after complete diagonalisation (with arrows indicating where the relevant eigenvalue originates from). The solid blue and dashed brown lines are the fitting curves (6.18) and (6.19), respectively. We have used the colour code blue diamonds $(m=2)$; brown circles $(m=3)$; orange triangles $(m=4)$; and green squares $(m=5)$.

\section{Discussion}

In the previous section we have studied the anomalous dimensions of the higher spin currents of the symmetric orbifold theory as one turns on the perturbation that corresponds to the string tension. While at the tensionless point, the higher spin fields of the Vasiliev theory - these correspond to the fields of the original $\mathcal{W}_{\infty}$ algebra - form a decoupled subsector, these fields begin to couple with the other stringy symmetry generators once the perturbation is switched on. As a consequence, the complete perturbation analysis is quite complicated. However, we have managed to solve it exactly for the first few values of the spin; we have also managed to obtain good analytic control over the diagonal entries of the mixing matrix, at least for the original higher spin generators - these are quadratic in the underlying free fields - and the generators that are cubic in the free fields. Our results are summarised in figure 1.

The results we have obtained seem to be very nicely in line with the idea that the original higher spin generators correspond to the 'leading' Regge trajectory, i.e., have the lowest mass (or anomalous dimension) for a given spin. We should stress that once mixing occurs (as will generically be the case), the actual eigenvectors will be linear combinations 
of fields from the different families (parametrised by $m$ ), see e.g., the explicit calculation for the $s=2$ field in section 6.2.3. However, it is legitimate to continue labelling the eigenvalues (and the associated eigenvectors) by the family from which they predominantly arise. In particular, the lowest eigenvalue will be associated to the family with the smallest diagonal entry in the mixing matrix. Our explicit calculations - we have also worked out some of the diagonal entries of the mixing matrix for the $m=4$ and $m=5$ families - suggest that the lowest eigenvalue of a given spin comes from the quadratic fields, i.e., that the extended $\mathcal{W}_{\infty}$ algebra (where we include also the generators arising from $(0 ;[2,0, \ldots, 0])$ and $(0 ;[0, \ldots, 0,2]))$ describes the 'leading Regge trajectory'.

By the same token, the cubic generators seem to describe the first sub-leading Regge trajectory. Furthermore, the dispersion relation, at least before any mixing is taken into account, seems to be of the form $E=s+a \log s$ (for some suitable value of $a$ ), which is what one should have expected for an AdS background. ${ }^{4}$ In particular, a dispersion relation of this kind was found, in a series of papers [7, 22-25], for slow-rotating long strings on $\mathrm{AdS}_{5} \times \mathrm{S}^{5}$, and the result was matched to a perturbative field theory calculation in [26-28], see [8] for a review. In the slow-rotating regime, the angular momentum on $S^{5}$ is negligible, and the result also directly applies to the $\mathrm{AdS}_{3}$ background that is of relevance to us. Actually, this conclusion is only valid for the $\mathrm{AdS}_{3}$ case with pure RR flux; for pure NSNS flux, it was shown in [9] that $\gamma(s)$ is instead constant, while for mixed NSNS/RR flux, the anomalous dimension also contains a $(\log (s))^{2}$ contribution [10]. We have also tried to fit our leading trajectory with an extra $(\log (s))^{2}$ term, and found the fitting coefficient of this term to be seven orders of magnitude smaller than the coefficient of the $\log (s)$ term. Thus our analysis gives credence to the belief that the symmetric orbifold theory is dual to string theory on $\mathrm{AdS}_{3}$ with pure RR flux.

The lifting of a specific class of states of the symmetric orbifold CFT was studied in $[29,30]$ within the context of the pp-wave/ $\mathrm{CFT}_{2}$ correspondence [31, 32]. The anomalous dimensions, computed to first order in the deformation parameter, were found to match the string spectrum to this order [30]. They considered states that contribute to the elliptic genus, i.e., that are chiral primaries on the right, and most of the interesting such states arise in the twisted sector of the symmetric orbifold. On the other hand, the higher spin currents we have considered in this paper have trivial right-moving conformal dimension and therefore all come from the untwisted sector. As a consequence, the two calculations are somewhat different in nature. We should also mention that, to the order to which we have done the calculation, there is no mixing between the two kinds of states since they have different bare conformal dimensions (their right-moving conformal dimensions differ).

The computations we have done in this paper apply fairly directly also to the symmetric orbifold of K3 [33], since the generators of the chiral algebra in that case form a subset of our generators, and the exactly marginal operators are the same as the ones we have used in our computation.

\footnotetext{
${ }^{4}$ We should mention that in higher dimensions, there are no additional symmetry generators beyond those of the Vasiliev theory in the large $N$ limit, and hence no mixing can occur. The corresponding calculations (including those for $\mathrm{AdS}_{3}$ ) therefore only determined the 'diagonal' matrix elements, without any diagonalisation.
} 
It would be very interesting to push this calculation further, and in particular solve the complete mixing problem up to some higher spins. This would allow us to determine the shape of the actual dispersion relations along the different Regge trajectories. It would also be very interesting to re-derive these anomalous dimensions from the dual AdS viewpoint. For the case of the $\mathcal{N}=3$ higher spin theory of [13] a bulk deformation computation was done for one spin 2 field in [34]. However, the field that switches on the string tension this is the preturbation we have considered in this paper - is not part of the perturbative higher spin theory; indeed, this follows from the identification of the twisted sector states with coset primaries, see eq. (3.2). Thus it is not clear how to rederive our result entirely within the framework of the higher spin theory.

\section{Acknowledgments}

We thank Niklas Beisert, Nikolay Bobev, Henriette Elvang, Rajesh Gopakumar, Matthew Headrick, Finn Larsen, Albion Lawrence and Wei Li for useful conversations. M.R.G. is grateful to Durham University for hospitality during the final stages of this work. C.P. acknowledges the hospitality from the Korea institute for Advanced Study, University of Michigan, Ann Arbor and Brandeis University at various stages of this work. The research of C.P. is supported by a grant of the Swiss National Science Foundation, while I.G.Z. is supported in part by the National Science Foundation under CAREER Grant No. PHY10-53842, and in part by the Department of Energy under Grant No. DE-SC0009987. This research was finally partly supported by the NCCR SwissMAP, funded by the Swiss National Science Foundation.

\section{A Free field realisation of the chiral fields}

Following on from the analysis of section 2, we describe in this appendix the fields at spin $s=\frac{3}{2}$, and also present the free field realisation of the stress-energy tensor $T$. Dropping for ease of notation the sum over the copies, we have

$$
\begin{array}{lll}
G^{++}=-2\left(: \partial \phi^{(2)} \bar{\psi}^{(1)}:+: \partial \bar{\phi}^{(1)} \psi^{(2)}:\right), & G^{++}=2\left(-: \partial \phi^{(2)} \bar{\psi}^{(1)}:+: \partial \bar{\phi}^{(1)} \psi^{(2)}:\right), \\
G^{--}=2\left(: \partial \phi^{(1)} \bar{\psi}^{(2)}:+: \partial \bar{\phi}^{(2)} \psi^{(1)}:\right), & & G^{--}=2\left(: \partial \phi^{(1)} \bar{\psi}^{(2)}:-: \partial \bar{\phi}^{(2)} \psi^{(1)}:\right) \\
G^{-+}=2\left(: \partial \phi^{(1)} \bar{\psi}^{(1)}:-: \partial \bar{\phi}^{(2)} \psi^{(2)}:\right), & G^{\prime-+}=2\left(: \partial \phi^{(1)} \bar{\psi}^{(1)}:+: \partial \bar{\phi}^{(2)} \psi^{(2)}:\right) \\
G^{+-}=2\left(-: \partial \phi^{(2)} \bar{\psi}^{(2)}:+: \partial \bar{\phi}^{(1)} \psi^{(1)}:\right), & G^{+-}=-2\left(: \partial \phi^{(2)} \bar{\psi}^{(2)}:+: \partial \bar{\phi}^{(1)} \psi^{(1)}:\right),
\end{array}
$$

all of which are elements of the $\mathcal{W}_{\infty}[0]$ algebra. The additional generators are the fields

$$
\begin{aligned}
C_{21} & =\sum_{a=1}^{N+1}: \psi_{a}^{(i)} \psi_{a}^{(j)} \bar{\psi}_{a}^{(k)}:, & C_{12} & =\sum_{a=1}^{N+1}: \psi_{a}^{(i)} \bar{\psi}_{a}^{(j)} \bar{\psi}_{a}^{(k)}:, \\
C_{20} & =\sum_{a=1}^{N+1}: \partial \phi_{a}^{(1)} \psi_{a}^{(2)}:, & C_{02} & =\sum_{a=1}^{N+1}: \partial \bar{\phi}_{a}^{(i)} \bar{\psi}_{a}^{(j)}: .
\end{aligned}
$$


The stress-energy tensor is of the form

$$
\begin{aligned}
T=\sum_{a=1}^{N+1}\{( & \left.: \partial \phi_{a}^{(1)} \bar{\partial} \phi_{a}^{(1)}:+: \partial \phi_{a}^{(2)} \bar{\partial} \phi_{a}^{(2)}:\right)+ \\
& \left.+\frac{1}{2}\left(: \partial \bar{\psi}_{a}^{(1)} \psi_{a}^{(1)}:-: \bar{\psi}_{a}^{(1)} \partial \psi_{a}^{(1)}:\right)+\frac{1}{2}\left(: \partial \bar{\psi}_{a}^{(2)} \psi_{a}^{(2)}:-: \bar{\psi}_{a}^{(2)} \partial \psi_{a}^{(2)}:\right)\right\} .
\end{aligned}
$$

For the $\mathcal{W}_{\infty}$ operators with higher spin we can work out the free field realisations by taking recursively OPEs of the above generators.

\section{B The second order analysis}

There are at least two natural approaches to calculate the integral (5.2), and we shall sketch them in the following.

\section{B.1 Using Stokes' theorem}

In this approach, the first step is to use the OPEs of the currents to rewrite the integrand of the first term on the right hand of (5.2)

$$
\begin{aligned}
& \sum_{m, n}\left(z_{1}-w_{1}\right)^{-h_{1}-m}\left(z_{2}-w_{2}\right)^{-h_{2}-n}\left\langle 0\left|\left(W_{m}^{1} \Phi^{1}\left(w_{1}, \bar{w}_{1}\right)\right)\left(W_{n}^{2} \Phi^{2}\left(w_{2}, \bar{w}_{2}\right)\right)\right| 0\right\rangle \\
& \quad+\sum_{m, n}\left(z_{1}-w_{2}\right)^{-h_{1}-m}\left(z_{2}-w_{1}\right)^{-h_{2}-n}\left\langle 0\left|\left(W_{m}^{1} \Phi^{2}\left(w_{2}, \bar{w}_{2}\right)\right)\left(W_{n}^{2} \Phi^{1}\left(w_{1}, \bar{w}_{1}\right)\right)\right| 0\right\rangle \\
& \quad+\sum_{m, n}\left(z_{1}-w_{1}\right)^{-h_{1}-m}\left(z_{2}-w_{1}\right)^{-h_{2}-n}\left\langle 0\left|\left(W_{m}^{1} W_{n}^{2} \Phi^{1}\left(w_{1}, \bar{w}_{1}\right)\right) \Phi^{2}\left(w_{2}, \bar{w}_{2}\right)\right| 0\right\rangle \\
& \quad+\sum_{m, n}\left(z_{1}-w_{1}\right)^{-h_{1}-m}\left(z_{1}-w_{1}\right)^{-h_{2}-n}\left\langle 0\left|\Phi^{2}\left(w_{2}, \bar{w}_{2}\right)\left(W_{m}^{1} W_{n}^{2} \Phi^{1}\left(w_{1}, \bar{w}_{1}\right)\right)\right| 0\right\rangle
\end{aligned}
$$

The $\bar{w}_{1}$ and $\bar{w}_{2}$ dependence is rather trivial - it equals $\frac{1}{\left(\bar{w}_{1}-\bar{w}_{2}\right)^{2}}-$ since only the perturbing fields have a non-trivial anti-chiral dependence. Thus each term above leads to an integral of the form

$$
\begin{array}{r}
\int d^{2} w_{1} d^{2} w_{2}\left(x_{1}-w_{1}\right)^{-p}\left(x_{2}-w_{2}\right)^{-q} \frac{\left\langle\mathcal{O}^{1}\left(w_{1}\right) \mathcal{O}^{2}\left(w_{2}\right)\right\rangle}{\left(\bar{w}_{1}-\bar{w}_{2}\right)^{2}} \\
=\int d^{2} w_{1}\left(x_{1}-w_{1}\right)^{-p} \int d^{2} w_{2} \frac{\partial_{\bar{w}_{2}}\left(\frac{\left\langle\mathcal{O}^{1}\left(w_{1}\right) \mathcal{O}^{2}\left(w_{2}\right)\right\rangle}{\left(\bar{w}_{1}-\bar{w}_{2}\right)}\right)}{\left(x_{2}-w_{2}\right)^{q}} \\
=\int d^{2} w_{1}\left(x_{1}-w_{1}\right)^{-p} \frac{1}{2 i} \oint_{x_{2}} d w_{2} \frac{\left(\frac{\left\langle\mathcal{O}^{1}\left(w_{1}\right) \mathcal{O}^{2}\left(w_{2}\right)\right\rangle}{\left(\bar{w}_{1}-\bar{w}_{2}\right)}\right)}{\left(x_{2}-w_{2}\right)^{q}}
\end{array}
$$


where we have used integration by parts. Now the contour integral can be evaluated by standard methods, and it yields

$$
\begin{aligned}
& =\frac{(-1)^{q} \pi}{(q-1) !} \int d^{2} w_{1} \frac{\left(x_{1}-w_{1}\right)^{-p}}{\left(\bar{w}_{1}-\bar{x}_{2}\right)}\left\langle\mathcal{O}^{1}\left(w_{1}\right)\left(\left(L_{-1}\right)^{q-1} \mathcal{O}^{2}\right)\left(x_{2}\right)\right\rangle \\
& =\frac{(-1)^{q} \pi}{(q-1) !} \int d^{2} w_{1} \frac{\partial_{\bar{w}_{1}}\left(\left\langle\mathcal{O}^{1}\left(w_{1}\right)\left(\left(L_{-1}\right)^{q-1} \mathcal{O}^{2}\right)\left(x_{2}\right)\right\rangle \log \left(\left|\bar{w}_{1}-\bar{x}_{2}\right|^{2}\right)\right)}{\left(x_{1}-w_{1}\right)^{p}} \\
& =\frac{(-1)^{q} \pi}{(q-1) ! 2 i} \oint_{x_{1}} d w_{1} \frac{\left\langle\mathcal{O}^{1}\left(w_{1}\right)\left(\left(L_{-1}\right)^{q-1} \mathcal{O}^{2}\right)\left(x_{2}\right)\right\rangle \log \left(\left|\bar{w}_{1}-\bar{x}_{2}\right|^{2}\right)}{\left(x_{1}-w_{1}\right)^{p}} \\
& =\frac{(-1)^{p+q} \pi^{2}}{(q-1) !(p-1) !}\left\langle\left(\left(L_{-1}\right)^{p-1} \mathcal{O}^{1}\right)\left(x_{1}\right)\left(\left(L_{-1}\right)^{q-1} \mathcal{O}^{2}\right)\left(x_{2}\right)\right\rangle \log \left(\left|\bar{x}_{1}-\bar{x}_{2}\right|^{2}\right),
\end{aligned}
$$

where, in the second line, we have used the identity

$$
\frac{1}{\bar{z}}=\partial_{\bar{z}} \log (z \bar{z}) \text {. }
$$

Note that the appearance of the factor $z \bar{z}$ is required to make the function single-valued. Applying this result to the expression (B.1) with $\Phi^{1}=\Phi^{2},{ }^{5}$ we find for the coefficient of $\pi^{2} \log \left(\left|\bar{z}_{1}-\bar{z}_{2}\right|^{2}\right)=2 \pi^{2} \log \left(\left|\bar{z}_{1}-\bar{z}_{2}\right|\right)$ the expression

$$
\begin{aligned}
& 2 \sum_{m, n} \frac{(-1)^{h_{1}+m+h_{2}+n-2}}{\left(h_{2}+n-1\right) !\left(h_{1}+m-1\right) !}\left\langle\left(\left(L_{-1}\right)^{h_{1}+m-1} W_{m}^{1} \Phi\right)\left(z_{1}\right)\left(\left(L_{-1}\right)^{h_{2}+n-1} W_{n}^{2} \Phi\right)\left(z_{2}\right)\right\rangle \\
& \quad=2\left\langle\left(\mathcal{N}\left(W^{1}\right)_{1} \Phi\right)\left(z_{1}\right)\left(\mathcal{N}\left(W^{2}\right)_{1} \Phi\right)\left(z_{2}\right)\right\rangle,
\end{aligned}
$$

where we have used the definition of (5.12), see also (4.1), and the $\mathcal{N}$ here is the corresponding vertex operators of the states. Including the overall normalisation factor then leads directly to eq. (5.6).

In order to obtain from this eq. (5.7), we recall that the 2-point function is related to the inner product of the associated state as

$$
\left\langle V\left(\psi_{2}, z_{2}\right) V\left(\psi_{1}, z_{1}\right)\right\rangle=(-1)^{h_{1}}\left\langle e^{-L_{1}} \psi_{2} \mid e^{-L_{1}} \psi_{1}\right\rangle\left(z_{2}-z_{1}\right)^{-h_{1}-h_{2}},
$$

which simplifies, for the case that both fields are quasi-primary, to the more familiar relation

$$
\left\langle V\left(\psi_{2}, z_{2}\right) V\left(\psi_{1}, z_{1}\right)\right\rangle=(-1)^{h_{1}}\left\langle\psi_{2} \mid \psi_{1}\right\rangle\left(z_{2}-z_{1}\right)^{-h_{1}-h_{2}} .
$$

A simple computation reveals that $L_{1} \mathcal{N}\left(W^{s}\right)_{1} \Phi=0$, and thus the 2-point function in (5.6) is, up to an irrelevant factor of $(-1)^{s}$, the norm of the two $\mathcal{N}\left(W^{s}\right)_{1} \Phi$ states.

To simplify the evaluation of the norm, we now move the $L_{1}$ 's in the bra-vector $\left\langle\mathcal{N}\left(W^{i}, \Phi\right)\right|$ to the right, where they annihilate the ket-vector $\left|\mathcal{N}\left(W^{s}\right)_{1} \Phi\right\rangle$; similarly we can move the $L_{-1}$ generators of $\left|\mathcal{N}\left(W^{s}\right)_{1} \Phi\right\rangle$ to the left. After a slightly tedious computation we then find

$$
\begin{aligned}
& \left\langle\mathcal{N}\left(W^{(s) i}, \Phi\right) \mathcal{N}\left(W^{(s) j}, \Phi\right)\right\rangle \\
& \quad=(-1)^{s-1} \sum_{m=1-s}^{s \bmod 1}(-1)^{\lceil s\rceil-1-\lfloor m\rfloor}\left(\begin{array}{c}
2 s-2 \\
s-1-m
\end{array}\right)\left\langle\Phi\left|W_{-m}^{(s) i} W_{m}^{(s) j}\right| \Phi\right\rangle .
\end{aligned}
$$

This is then eq. (5.7).

\footnotetext{
${ }^{5}$ The last two lines do not give rise to logarithmic terms since either $p$ or $q$ is zero; they therefore do not play a role for our computation.
} 


\section{B.2 Using separation of variables}

The second approach differs from the above in the treatment of the integral (B.2). The idea of the computation is to split off from the double integral the UV divergence, so that the remainder is UV finite (and contains the anomalous dimension). More concretely, we use the Möbius symmetry to rewrite the 4-point function in the integrand as

$$
\begin{aligned}
& \left\langle W^{i}\left(z_{1}+\frac{\epsilon\left(z_{1}-z_{4}\right)\left(z_{1}-z_{2}\right)}{\left(z_{4}-z_{2}\right)}\right) \Phi\left(z_{2}\right) \Phi\left(z_{3}\right) W^{j}\left(z_{4}\right)\right\rangle \\
& =\left(z_{4}-z_{1}\right)^{-2 s_{i}}\left|\frac{\left(z_{1}-z_{4}\right)^{2}}{\left(z_{3}-z_{1}\right)^{2}\left(z_{2}-z_{4}\right)^{2}}\right|^{2}\left\langle W^{i}\left(\epsilon^{-1}\right) \Phi(1) \Phi(x) W^{j}(0)\right\rangle,
\end{aligned}
$$

where now the integration variables are $z_{2}$ and $x$. Because of the change of variables, there is a Jacobian factor

$$
\left|d z_{2}\right|^{2}\left|d z_{3}\right|^{2}=\left|d z_{2}\right|^{2}|d x|^{2}\left|\frac{\partial\left(z_{2}, z_{3}\right)}{\partial\left(z_{2}, x\right)}\right|^{2},
$$

and the original integral becomes

$$
\begin{aligned}
& \iint\left|d z_{2}\right|^{2}\left|d z_{3}\right|^{2}\left\langle W^{i}\left(z_{1}+\epsilon\right) \Phi\left(z_{2}\right) \Phi\left(z_{3}\right) W^{j}\left(z_{4}\right)\right\rangle \\
& =\int\left|d z_{2}\right|^{2}|d x|^{2}\left(z_{4}-z_{1}\right)^{-2 s_{i}}\left|\frac{\left(z_{1}-z_{4}\right)}{\left(z_{1}-z_{2}\right)\left(z_{2}-z_{4}\right)}\right|^{2}\left\langle W^{i}\left(\epsilon^{-1}\right) \Phi(1) \Phi(x) W^{j}(0)\right\rangle .
\end{aligned}
$$

Carrying out the $z_{2}$ integration, we now get

$$
2 \pi \log \left(\Lambda^{2}\left|z_{4}-z_{1}\right|^{2}\right)\left(z_{4}-z_{1}\right)^{-2 s_{i}} \int|d x|^{2}\left\langle W^{i}\left(\epsilon^{-1}\right) \Phi(1) \Phi(x) W^{j}(0)\right\rangle,
$$

where $\Lambda$ is a UV cutoff. It remains to do the $d x$ integral. Since the left- and right-moving parts of the correlation function decouple, we can rewrite (B.14) as

$$
\left\langle W^{i}\left(\epsilon^{-1}\right) \Phi(1,1) \Phi(x, \bar{x}) W^{j}(0)\right\rangle=\frac{1}{(1-\bar{x})^{2}}\left\langle W^{i}\left(\epsilon^{-1}\right) \Phi(1) \Phi(x) W^{j}(0)\right\rangle .
$$

As in (B.3) above, this integral now becomes

$$
\oint_{C} \frac{d x}{2 i} \frac{1}{(1-\bar{x})}\left\langle W^{i}\left(\epsilon^{-1}\right) \Phi(1) \Phi(x) W^{j}(0)\right\rangle,
$$

where $C$ is a contour that circles around all the insertion points. The integrand reads

$$
\begin{aligned}
\left\langle W^{i}\left(\epsilon^{-1}\right) \Phi(1) \Phi(x) W^{j}(0)\right\rangle= & \left\langle 0\left|W_{h_{i}}^{i} \Phi(1) \Phi(x) W_{-h_{i}}^{j}\right| 0\right\rangle \\
= & \left\langle 0\left|\left[W_{h_{i}}^{i}, \Phi(1)\right] \Phi(x) W_{-h_{i}}^{j}\right| 0\right\rangle+\left\langle 0\left|\Phi(1)\left[W_{h_{i}}^{i}, \Phi(x)\right] W_{-h_{i}}^{j}\right| 0\right\rangle \\
& +\left\langle 0\left|\Phi(1) \Phi(x)\left[W_{h_{i}}^{i}, W_{-h_{i}}^{j}\right]\right| 0\right\rangle,
\end{aligned}
$$

where the third term is the disconnected part of the 4-point function that is subtracted out in the second line of (5.2). The first two terms can be evaluated using, e.g., the 
techniques of [35]

$$
\begin{aligned}
= & \left\langle 0\left|\sum_{m=1-h_{i}}^{h_{i}}\left(\begin{array}{c}
2 h_{i}-1 \\
m+h_{i}-1
\end{array}\right)\left(W_{m}^{i} \Phi\right)(1) \Phi(x) W_{-h_{i}}^{j}\right| 0\right\rangle \\
& +\left\langle 0\left|\Phi(1) \sum_{p=1-h_{i}}^{h_{i}}\left(\begin{array}{c}
2 h_{i}-1 \\
m+h_{i}-1
\end{array}\right) x^{h_{i}-m}\left(W_{m}^{i} \Phi\right) W_{-h_{i}}^{j}\right| 0\right\rangle .
\end{aligned}
$$

Repeating the same step then finally leads to

$$
\begin{aligned}
= & \sum_{m=1-h_{i}}^{h_{i}} \sum_{n=1-h_{j}}^{\infty}\left(\begin{array}{c}
2 h_{i}-1 \\
m+h_{i}-1
\end{array}\right)(-1)^{n+h_{j}-1} \frac{(-1)^{1}}{(1-x)^{1-m-n+1}}\left\langle e^{-L_{1}} W_{n}^{j} W_{m}^{i} \Phi \mid \Phi\right\rangle \\
+ & \sum_{m=1-h_{i}}^{h_{i}} \sum_{n=1-h_{j}}^{\infty}\left(\begin{array}{c}
h_{j}+n-1 \\
h_{j}-h_{j}
\end{array}\right)(-1)^{n+h_{j}-1}(x)^{-n-h_{j}}\left(\begin{array}{c}
2 h_{i}-1 \\
m+h_{i}-1
\end{array}\right) \\
& \frac{(-1)^{1-n}}{(1-x)^{1-m+1-n}}\left\langle e^{-L_{1}} W_{m}^{i} \Phi \mid e^{-L_{1}} W_{n}^{j} \Phi\right\rangle,
\end{aligned}
$$

where we have used (B.6). In the first line, the only $x$-dependence appears in the $1-x$ pole which will not contribute to the contour integral. Thus only the second and third line contribute, and the contour integral (B.15) becomes

$$
\begin{gathered}
\oint \frac{d x}{2 i} \sum_{m=1-h_{i}}^{h_{i}} \sum_{n=1-h_{j}}^{\infty}\left(\begin{array}{c}
h_{j}+n-1 \\
h_{j}-h_{j}
\end{array}\right)\left(\begin{array}{c}
2 h_{i}-1 \\
m+h_{i}-1
\end{array}\right) \frac{(-1)^{h_{j}}}{(x)^{n+h_{j}}} \frac{\left\langle e^{-L_{1}} W_{m}^{i} \Phi \mid e^{-L_{1}} W_{n}^{j} \Phi\right\rangle}{(1-x)^{1-m+1-n}} \\
=\pi \sum_{m=1-h_{i}}^{h_{i}} \sum_{n=1-h_{j}}^{\infty}\left(\begin{array}{c}
2 h_{i}-1 \\
m+h_{i}-1
\end{array}\right)\left(\begin{array}{c}
-m+h_{j} \\
n+h_{j}-1
\end{array}\right)(-1)^{h_{j}}\left\langle e^{-L_{1}} W_{m}^{i} \Phi \mid e^{-L_{1}} W_{n}^{j} \Phi\right\rangle .
\end{gathered}
$$

Rearranging the $L_{ \pm 1}$ as in the previous subsection

$$
\begin{aligned}
& \left\langle e^{-L_{1}} W_{m}^{i} \Phi \mid e^{-L_{1}} W_{n}^{j} \Phi\right\rangle \\
& \quad=\sum_{p=0}^{-\lfloor m\rfloor}(-1)^{m-n}\left(\begin{array}{c}
h_{i}-1-m \\
p
\end{array}\right)\left(\begin{array}{c}
h_{j}-1-n \\
p+m-n
\end{array}\right)\left\langle W_{p+m}^{i} \Phi \mid W_{p+m}^{j} \Phi\right\rangle,
\end{aligned}
$$

eq. (B.17) can be rewritten as

$$
=(-1)^{s-1} \sum_{m=1-s}^{s \bmod 1}(-1)^{\lceil s\rceil-1-\lfloor m\rfloor}\left(\begin{array}{c}
2 s-2 \\
s-1-m
\end{array}\right)\left\langle\Phi\left|W_{-m}^{(s) i} W_{m}^{(s) j}\right| \Phi\right\rangle,
$$

which agrees precisely with eq. (B.9). ${ }^{6}$

\footnotetext{
${ }^{6}$ In the case when the currents are fermions, we have picked the branch of the square-root (due to halfinteger spin) such that the resulting anomalous dimensions have the same sign as the anomalous dimensions of the bosons in the same supermultiplet. Notice that there is no such difficulty in the computation of the anomalous dimensions of the bosonic fields.
} 
Open Access. This article is distributed under the terms of the Creative Commons Attribution License (CC-BY 4.0), which permits any use, distribution and reproduction in any medium, provided the original author(s) and source are credited.

\section{References}

[1] M.R. Gaberdiel and R. Gopakumar, Higher Spins \& Strings, JHEP 11 (2014) 044 [arXiv: 1406.6103] [INSPIRE].

[2] M.R. Gaberdiel and R. Gopakumar, Large $N=4$ Holography, JHEP 09 (2013) 036 [arXiv: 1305.4181] [INSPIRE].

[3] M.A. Vasiliev, Nonlinear equations for symmetric massless higher spin fields in (A) $d S_{d}$, Phys. Lett. B 567 (2003) 139 [hep-th/0304049] [INSPIRE].

[4] S.F. Prokushkin and M.A. Vasiliev, Higher spin gauge interactions for massive matter fields in 3 -D AdS space-time, Nucl. Phys. B 545 (1999) 385 [hep-th/9806236] [INSPIRE].

[5] S. Prokushkin and M.A. Vasiliev, 3-D higher spin gauge theories with matter, hep-th/9812242 [INSPIRE].

[6] M.R. Gaberdiel and R. Gopakumar, Stringy Symmetries and the Higher Spin Square, J. Phys. A 48 (2015) 185402 [arXiv:1501.07236] [InSPIRE].

[7] S.S. Gubser, I.R. Klebanov and A.M. Polyakov, A semiclassical limit of the gauge/string correspondence, Nucl. Phys. B 636 (2002) 99 [hep-th/0204051] [INSPIRE].

[8] N. Beisert et al., Review of AdS/CFT Integrability: An overview, Lett. Math. Phys. 99 (2012) 3 [arXiv: 1012.3982] [INSPIRE].

[9] A. Loewy and Y. Oz, Large spin strings in AdS $S_{3}$, Phys. Lett. B 557 (2003) 253 [hep-th/0212147] [INSPIRE].

[10] J.R. David and A. Sadhukhan, Spinning strings and minimal surfaces in $A d S_{3}$ with mixed 3-form fluxes, JHEP 10 (2014) 49 [arXiv:1405.2687] [INSPIRE].

[11] T. Creutzig, Y. Hikida and P.B. Ronne, Extended higher spin holography and Grassmannian models, JHEP 11 (2013) 038 [arXiv:1306.0466] [INSPIRE].

[12] C. Candu and C. Vollenweider, On the coset duals of extended higher spin theories, JHEP 04 (2014) 145 [arXiv:1312.5240] [INSPIRE].

[13] T. Creutzig, Y. Hikida and P.B. Ronne, Higher spin AdS $S_{3}$ holography with extended supersymmetry, JHEP 10 (2014) 163 [arXiv:1406.1521] [INSPIRE].

[14] C. Candu, C. Peng and C. Vollenweider, Extended supersymmetry in AdS $S_{3}$ higher spin theories, JHEP 12 (2014) 113 [arXiv:1408.5144] [INSPIRE].

[15] J.R. David, G. Mandal and S.R. Wadia, Microscopic formulation of black holes in string theory, Phys. Rept. 369 (2002) 549 [hep-th/0203048] [INSPIRE].

[16] M.R. Gaberdiel, K. Jin and W. Li, Perturbations of W(infinity) CFTs, JHEP 10 (2013) 162 [arXiv: 1307.4087] [INSPIRE].

[17] J.L. Cardy, Conformal Invariance and Statistical Mechanics, in Les Houches XLIX (1988), Fields, Strings and Critical Phenomena, E. Brezin and J. Zinn-Justin eds., North-Holland, (1990), pg. 169-246. 
[18] S. Fredenhagen, M.R. Gaberdiel and C.A. Keller, Symmetries of perturbed conformal field theories, J. Phys. A 40 (2007) 13685 [arXiv:0707.2511] [InSPIRE].

[19] M.R. Gaberdiel and C. Peng, The symmetry of large $\mathcal{N}=4$ holography, JHEP 05 (2014) 152 [arXiv: 1403.2396] [INSPIRE].

[20] O. Aharony, A.B. Clark and A. Karch, The CFT/AdS correspondence, massive gravitons and a connectivity index conjecture, Phys. Rev. D 74 (2006) 086006 [hep-th/0608089] [INSPIRE].

[21] O. Aharony, S.S. Gubser, J.M. Maldacena, H. Ooguri and Y. Oz, Large-N field theories, string theory and gravity, Phys. Rept. 323 (2000) 183 [hep-th/9905111] [INSPIRE].

[22] S. Frolov and A.A. Tseytlin, Semiclassical quantization of rotating superstring in $A d S_{5} \times S^{5}$, JHEP 06 (2002) 007 [hep-th/0204226] [INSPIRE].

[23] R. Roiban, A. Tirziu and A.A. Tseytlin, Two-loop world-sheet corrections in $A d S_{5} \times S^{5}$ superstring, JHEP 07 (2007) 056 [arXiv:0704.3638] [INSPIRE].

[24] R. Roiban and A.A. Tseytlin, Strong-coupling expansion of cusp anomaly from quantum superstring, JHEP 11 (2007) 016 [arXiv:0709.0681] [INSPIRE].

[25] S. Giombi, R. Ricci, R. Roiban, A.A. Tseytlin and C. Vergu, Quantum $A d S_{5} \times S^{5}$ superstring in the AdS light-cone gauge, JHEP 03 (2010) 003 [arXiv:0912.5105] [INSPIRE].

[26] A.V. Belitsky, A.S. Gorsky and G.P. Korchemsky, Logarithmic scaling in gauge/string correspondence, Nucl. Phys. B 748 (2006) 24 [hep-th/0601112] [InSPIRE].

[27] B. Eden and M. Staudacher, Integrability and transcendentality, J. Stat. Mech. 0611 (2006) P11014 [hep-th/0603157] [INSPIRE].

[28] N. Beisert, B. Eden and M. Staudacher, Transcendentality and Crossing, J. Stat. Mech. 0701 (2007) P01021 [hep-th/0610251] [INSPIRE].

[29] J. Gomis, L. Motl and A. Strominger, PP wave/CFT 2 duality, JHEP 11 (2002) 016 [hep-th/0206166] [INSPIRE].

[30] E. Gava and K.S. Narain, Proving the PP wave/CFT 2 duality, JHEP 12 (2002) 023 [hep-th/0208081] [INSPIRE].

[31] Y. Hikida and Y. Sugawara, Superstrings on PP wave backgrounds and symmetric orbifolds, JHEP 06 (2002) 037 [hep-th/0205200] [INSPIRE].

[32] O. Lunin and S.D. Mathur, Rotating deformations of $A d S_{3} \times S^{3}$, the orbifold CFT and strings in the pp wave limit, Nucl. Phys. B 642 (2002) 91 [hep-th/0206107] [INSPIRE].

[33] M. Baggio, M.R. Gaberdiel and C. Peng, Higher spins in the symmetric orbifold of K3, Phys. Rev. D 92 (2015) 026007 [arXiv: 1504.00926] [INSPIRE].

[34] Y. Hikida and P.B. Rønne, Marginal deformations and the Higgs phenomenon in higher spin $A d S_{3}$ holography, JHEP 07 (2015) 125 [arXiv: 1503.03870] [INSPIRE].

[35] M. Gaberdiel, Fusion rules of chiral algebras, Nucl. Phys. B 417 (1994) 130 [hep-th/9309105] [INSPIRE]. 